\title{
Primary skeletal muscle diffuse large B cell lymphoma: A case report and review of the literature
}

\author{
LIJUAN ZHANG ${ }^{1}$, QUANDE LIN ${ }^{2}$, LINA ZHANG ${ }^{2}$, LIHUA DONG ${ }^{2}$ and YUFU LI ${ }^{2}$ \\ ${ }^{1}$ Transplantation Department of Internal Medicine, 302 Hospital of the Chinese People's Liberation Army, Beijing 100039; \\ ${ }^{2}$ Department of Hematology, Affiliated Cancer Hospital of Zhengzhou University, Zhengzhou, Henan 450008, P.R. China
}

Received September 7, 2014; Accepted June 2, 2015

DOI: $10.3892 / \mathrm{ol} .2015 .3505$

\begin{abstract}
The occurrence of primary extranodal non-Hodgkin's lymphoma (NHL) of soft tissue is rare, particularly in skeletal muscle. The present study describes a case of diffuse large B cell lymphoma of the right lower extremity and provides a detailed review of the literature associated with this disorder, with the aim of improving the future diagnosis and therapy of extranodal NHL. The present case report was of a 76-year-old woman who presented with a right thigh and calf mass. In view of the tumor's location and the patient's age, soft tissue tumors were considered to be soft tissue sarcoma. Imaging scans were performed to determine the location and size of the tumor, followed by a biopsy of the muscle. Histopathological examination then yielded a diagnosis of diffuse large B cell lymphoma. The patient then underwent 4 cycles of chemotherapy. There was evident relief of pain and swelling in the right extremity; however, positron emission tomography/computed tomography (PET/CT) determined insufficient treatment efficacy. Chemotherapy was adjusted for 2 cycles; however, the patient suffered an aggravation of edema, so a different chemotherapy regimen of bleomycin, cytarabine, vincristine, cyclosphamide and dexamethasone (BCOAD) was performed for a further 2 cycles. The edema was alleviated and magnetic resonance imaging revealed shrinkage of the lower limb mass and the right thigh mass was undetectable. In conclusion, the present case report demonstrated that PET/CT may help determine the efficacy of chemotherapy treatment and that the BCOAD chemotherapy regimen may be more effective than standard treatments in certain cases.
\end{abstract}

Correspondence to: Dr Yufu Li, Department of Hematology, Affiliated Cancer Hospital of Zhengzhou University, 127 Dongming Road, Zhengzhou, Henan 450008, P.R. China E-mail: yufu_li85@163.com

Key words: extranodal lymphoma, diffuse large B cell lymphoma, skeletal muscle, therapy

\section{Introduction}

Diffuse large B cell lymphoma (DLBCL) is the most prevalent form of non-Hodgkin's lymphoma (NHL) and has a high incidence rate among elderly people $(1,2)$. The extranodal type of NHL is identified in $20-30 \%$ of patients (3). DLBCL often presents in extranodal sites, including the testis, skin, lung, bone and central nervous system, as well as the respiratory and gastrointestinal tracts (4). Primary skeletal muscle lymphoma is exceptionally rare form of DLBCL, particularly in the thigh and calf areas. The main symptoms of the disease typically include presence of a mass, pain and swelling (5). Imaging is conducted in order to establish a diagnosis, and the most common imaging techniques used include positron emission tomography/computed tomography (PET/CT) and magnetic resonance imaging (MRI). The present study reports a case of primary skeletal muscle lymphoma and provides a detailed review of the literature associated with this disorder.

\section{Case report}

In March 2013, a 76-year-old female patient was admitted to the Affiliated Cancer Hospital of Zhengzhou University (Zhengzhou, China) and presented with a 1-year history of right foot numbness, which gradually worsened. In January 2013, the patient began experiencing pain and swelling in the right thigh and calf. However, the patient denied suffering from typical 'B' symptoms, including fever, night sweats and weight loss, and had no history of tobacco smoking, alcohol consumption or drug use.

Patient examination revealed a palpable firm mass of $54 \times 48 \mathrm{~mm}$ in the right thigh and calf muscle, as well as several 10-28 $\mathrm{mm}$ firm, tender and mobile nodules distributed over the patients right groin and popliteal fossa. However, there was no evidence of hepatosplenomegaly. In addition, the patient had an Eastern Cooperative Oncology Group performance score of 2 (http://ecog-acrin.org/resources/ecog-performance-status).

The blood test results were as follows: Hemoglobin, $10.5 \mathrm{~g} / \mathrm{dl}$ (normal range, 12-16 g/dl); white cell count, 5.89x10 $/ 1$; platelets, $268 \times 10^{9} / 1$; lactate dehydrogenase, $466 \mathrm{U} / 1$ (normal range, 109-245 U/1); and $\beta 2$-microglobulin, $3.81 \mathrm{mg} / \mathrm{l}$ (normal range, $0.8-2.2 \mathrm{mg} / \mathrm{l})$. Serological tests for hepatitis B and C virus as well as human immunodeficiency virus were negative. MRI with enhanced T1-weighted sequence revealed a hyperintense 


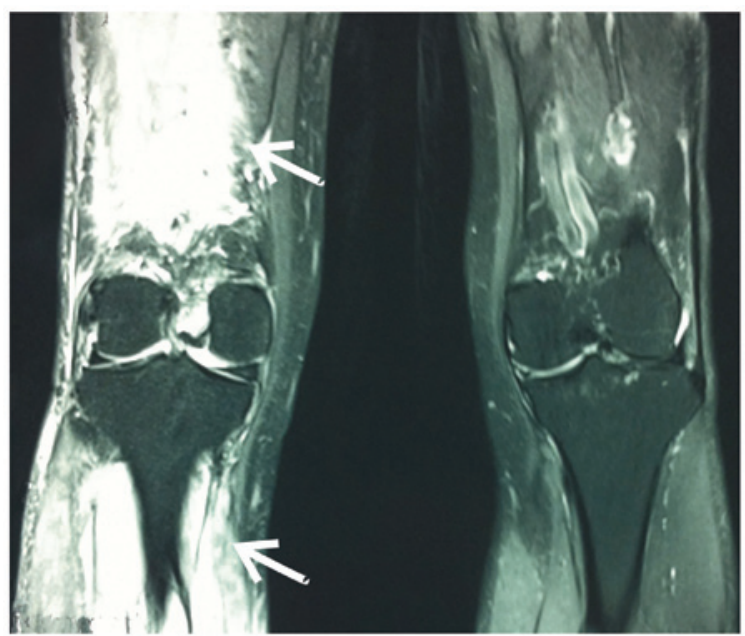

Figure 1. Features of the enhanced T1-weighted magnetic resonance imaging scans. Coronal section revealed a bulky uniform distension of skeletal muscles, which was hyperintense relative to normal muscle.
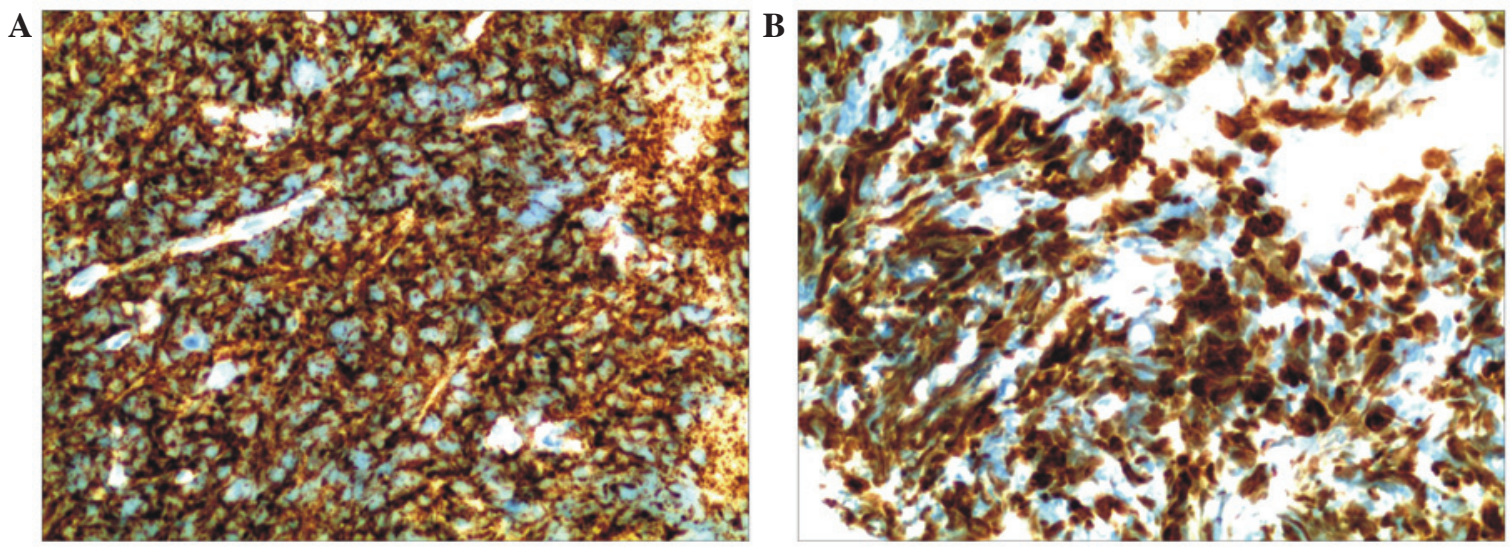

Figure 2. Immunohistochemistry of the patient. Immunohistochemical detection of (A) CD20 and (B) Ki-67 (magnification, x200).

mass in the lower tibia and upper fibula (Fig. 1), 90x54x48 mm in size. Enlarged lymph nodes were observed in the right popliteal, right inguinal and right iliac artery, 20-40 $\mathrm{mm}$ in size. CT scans revealed enlarged lymph nodes in the mediastinum, while the bilateral pulmonary region was normal. Bone marrow aspiration results were normal. A muscle biopsy was performed and the histological analysis revealed a diagnosis of primary skeletal muscle NHL DLBCL. Immunostaining of the tumoral cells demonstrated positive staining for CD20 and CD79 $\alpha$, which is typical of cells with B phenotype (Fig. 2A). Malignant cells were strongly positive for Ki-67 (70\%) (Fig. 2B). In addition, CD3, CD43, epithelial membrane antigen, CD56 and synaptophysin were negative in the malignant cells. The patient was diagnosed as DLBCL stage IV, with an international prognostic index score of 4 , indicating high risk (6).

The patient received four cycles (21 days each) of R-CHOP chemotherapy, which involved administration of the following: $375 \mathrm{mg} / \mathrm{m}^{2}$ rituximab over $6 \mathrm{~h}$ on day $-1 ; 750 \mathrm{mg} / \mathrm{m}^{2}$ cyclophosphamide, $2 \mathrm{mg}$ vincristine and $50 \mathrm{mg} / \mathrm{m}^{2}$ doxorubicin (intravenous) on day 1 ; and $40 \mathrm{mg} / \mathrm{m}^{2}$ prednisolone (orally) on days $1-5$, every 21 -day cycle. Chemotherapy was administered intravenously once a month for a total of four cycles. The patient suffered neutropenia (absolute neutrophil count, $<0.5 \times 10^{9} / 1$ ) and infectious complication during the first cycle. The patient was then treated with a modified R-Mini-CHOP (day 1, $600 \mathrm{mg} / \mathrm{m}^{2}$ cyclophosphamide, $2 \mathrm{mg} / \mathrm{m}^{2}$ vindesine and $375 \mathrm{mg} / \mathrm{m}^{2}$ rituximab; day $2,50 \mathrm{mg} / \mathrm{m}^{2}$ epirubicin; and days $1-5$, $40 \mathrm{mg} / \mathrm{m}^{2}$ prednisone) for the remaining three cycles. There was evident relief of pain and swelling in the right extremity. PET/CT imaging with 18F-fluorodeoxyglucose revealed a reduced fluorodeoxyglucose (FDG) uptake in the tumor and lymph nodes. However, in addition to the right thigh and calf muscle, FDG uptake persisted in the right iliac artery lymph nodes and right inguinal lymph nodes (Fig. 3A). PET/CT was used to evaluate the efficacy of the treatment, which revealed a stabilization of the disease. The patient was further treated with chemotherapy as follows: Day $1,375 \mathrm{mg} / \mathrm{m}^{2}$ rituximab; day $3 / 7,1000 \mathrm{mg} / \mathrm{m}^{2}$ gemcitabine; day $3-7,15 \mathrm{mg} / \mathrm{m}^{2}$ cisplatin; and day 3-7, $9 \mathrm{mg}$ dexamethasone (R-GDP) every 30 days for a total of 2 cycles. In October 2013 (2 months after chemotherapy), the patient suffered a gradual aggravation of edema, swelling and pain in the right lower limb. Doppler ultrasound results revealed an enlarged hypoechoic mass in the right calf, $72 \times 43 \mathrm{~mm}$ in size. Subsequently, an adjusted chemotherapy regimen of bleomycin, cytarabine, vincristine, cyclosphamide and dexamethasone (BCOAD) regimen was administrated 
A

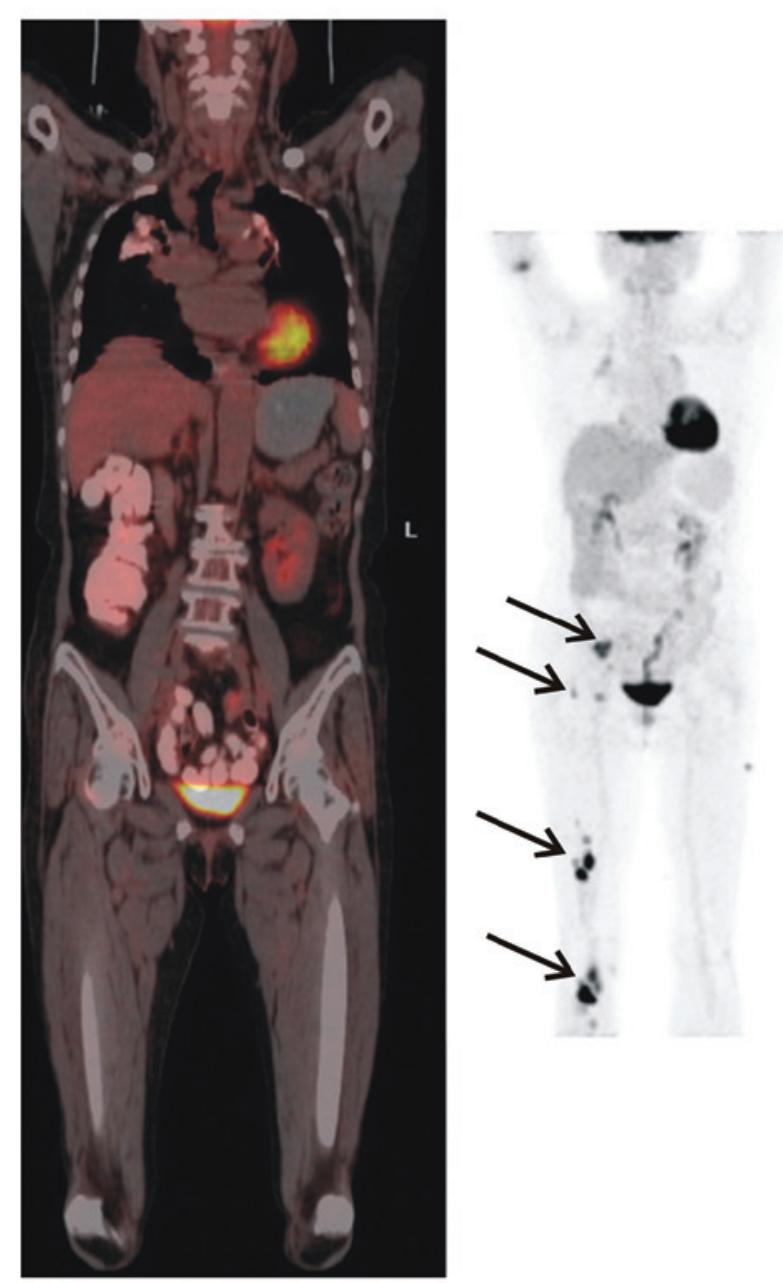

B

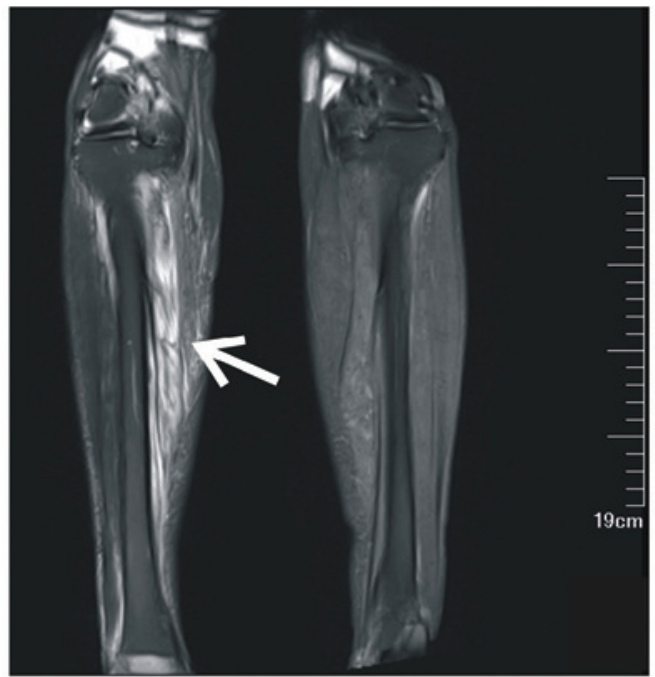

Figure 3. Imaging of the patient following chemotherapy. (A) FDG positron emission tomography/computerized tomography of the muscle following 4 cycles of chemotherapy demonstrated striking FDG uptake involving the skeletal muscles of the right thigh and calf muscle as well as the right iliac artery and right inguinal lymph nodes (arrows). (B) Coronal enhanced T1-weighted magnetic resonance imaging of the muscle following 8 cycles of chemotherapy. Coronal section revealed a diffuse, relatively homogenous hyperintense mass (arrow) in right calf muscles. FDG, fluorodeoxyglucose.

for 2 cycles. Subsequently, the patient received two cycles of R-BCOAD chemotherapy, which involved administration of the following: $375 \mathrm{mg} / \mathrm{m}^{2}$ rituximab over $6 \mathrm{~h}$ on day -1 ; $2.5 \mathrm{mg} / \mathrm{m}^{2}$ bleomycin, $0.5 \mathrm{mg}$ vincristine and $10 \mathrm{mg} / \mathrm{m}^{2}$ cytarabine continuous infusion over $24 \mathrm{~h}$ on days $1-4 ; 650 \mathrm{mg} / \mathrm{m}^{2}$ cyclophosphamide on day 5 ; and $10 \mathrm{mg} / \mathrm{m}^{2}$ prednisolone (orally) on days $1-5$, every 21 -day cycle. This chemotherapy was administered through a continuous intravenous infusion of bleomycin, cytarabine and vincristine over $96 \mathrm{~h}$. The edema was completely alleviated and the mass in the right lower limb began to shrink. MRI results revealed a reduction in tumor size in the lower limb and the mass in the right thigh became undetectable (Fig. 3B). The patient received a local radiation therapy; however, the effect of the treatment was poor and the mass gradually increased. The patient succumbed to the disease on July 2, 2014. Written informed consent was obtained from the patient's family prior to the publication of the present study.

\section{Discussion}

Primary skeletal muscle lymphoma was first reported by Kandel et al (7) in 1984 and has since been reported to account for $\sim 0.5 \%$ of extranodal lymphomas (8), with an incidence rate of $<0.1 \%$ in all lymphoma of the extremities (9). Therefore, primary skeletal muscle NHL of diffuse large B cell immunophenotype is exceptionally rare. This disease may occur through one of the three ways: As disseminated disease via the hematogenous or lymphatic pathways; as an extension from adjacent organs, such as bones or lymph nodes; or very rarely, as primary extranodal disease (10).

The common clinical symptoms of primary skeletal muscle lymphoma are usually associated with muscle swelling, limb pain and edema, without any sign of heat and redness (5); in addition, this disease may occur as isolated lesions (11). The clinical features of the extranodal lymphoma include pain and tenderness, lymphadenopathy, ipsilateral extremity swelling and elevated lactate dehydrogenase, which therefore aid the diagnosis the primary skeletal muscle lymphoma (12). Furthermore, primary skeletal muscle lymphoma exhibits certain distinctive MRI features, which allow it to be differentiated from other types of soft-tissue tumors: On T1-weighted images, an increased signal intensity is commonly observed compared with normal muscle; and on T2-weighted images, intermediate signal intensity is observed compared with fat $(11,13)$. In addition, on contrast-enhanced MRI, skeletal 
muscle lymphoma demonstrates homogeneous diffuse enhancement (14). CT scans may reveal muscle swelling and also serve as a tool to guide biopsy. With the development of technology, the clinical application of PET/CT has become increasingly important for lymphoma diagnosis and tumor staging (15). In the present study, PET/CT was employed in order to assess the efficacy of the chemotherapy treatments. However, imaging features of lymphoma in skeletal muscle are nonspecific and it may be difficult to distinguish lymphoma from other diseases, such as primary soft tissue, sarcoma, metastases, trauma or myositis (11). Therefore, biopsy and pathological evaluation are essential for the diagnosis of primary skeletal lymphoma (16). The present case report illustrated that MRI and CT provided the basis for diagnosis and that the diagnosis was confirmed through biopsy histopathology and immunohistochemistry.

The treatment of primary skeletal muscle lymphoma relies predominantly on the type of lymphoma. The prognosis of primary skeletal muscle lymphoma is poor compared with that of lymph node lymphoma, especially at stages III-IV. Therefore, selecting the most effective treatment regimen is essential. The present study reported a case of DLBCL, the standard treatment for which is R-CHOP (17-21).

The combination of chemotherapy and radiotherapy significantly was reported to increase disease-free survival and overall survival (OS) rates (22). In addition, chemotherapy followed by local radiotherapy, compared with chemotherapy alone, demonstrated improved event-free survival (EFS) results (23). A previous study reported 5-year survival, EFS and OS rates of 94,84 and $91 \%$, respectively (24). However, $50 \%$ of DLBCL patients are unresponsive to the standard chemotherapy or suffer disease relapse $(19,20)$. Patients with refractory NHL have limited treatment options and poor prognosis. Hou et al (25) reported that GDP with or without rituximab was effective in patients with refractory or relapsed aggressive B cell NHL. The overall response rate of patients with recurrent history or patients with refractory aggressive histology B cell NHL is $49-72 \%$ (25-27). In addition, $28 \%$ of patients suffered grade III-IV neutropenia and $40 \%$ of patients suffered thrombocytopenia (25-27). Aribi et al (26) reported that the 3-year progression-free and EFS rates were $20.5 \%$ (range, 16.3-24\%) and $19.7 \%$ (range, 15.9-23.5\%) for the GPD regimen, respectively. These results indicated that GDP with or without rituximab may be a promising treatment option for refractory DLBCL. However, in the present study, the patient received a modified BCOAD regimen, which had not been previously reported; the results demonstrated that the treatment was effective in this patient and to date the patient has remained stable and healthy.

In conclusion, primary skeletal muscular DLBCL is a rare lesion that frequently occurs among elderly adults. Imaging can be used to reveal the location and the size of the mass. Biopsy or surgery are the key to the diagnosis of DLBCL. Selection of the appropriate treatment regimen is challenging and requires further investigation.

\section{References}

1. Yancik R and Ries LA: Cancer in older persons: An international issue in an aging world. Semin Oncol 31: 128-136, 2004.
2. Flowers CR, Sinha R and Vose JM: Improving outcomes for patients with diffuse large B-cell lymphoma. CA Cancer J Clin 60: 393-408, 2010.

3. Freeman C, Berg JW and Cutler SJ: Occurrence and prognosis of extranodal lymphomas. Cancer 29: 252-260, 1972.

4. Bourdeanu L, Menon R and Somlo G: Diffuse large B-cell lymphoma with calf muscle localization. Case Rep Hematol 2011: 292494, 2011

5. Lee VS, Martinez S and Coleman RE: Primary muscle lymphoma: Clinical and imaging findings. Radiology 203: 237-244, 1997.

6. Talaulikar D, Shadbolt B, Dahlstrom JE and McDonald A Routine use of ancillary investigations in staging diffuse large B-cell lymphoma improves the International Prognostic Index (IPI). J Hematol Oncol 2: 49, 2009.

7. Kandel RA, Bédard YC, Pritzker KP and Luk SC: Lymphoma. Presenting as an intramuscular small cell malignant tumor. Cancer 53: 1586-1589, 1984.

8. Glass AG, Karnell LH and Menck HR: The National Cancer Data Base report on non-Hodgkin's lymphoma. Cancer 80: 2311-2320, 1997.

9. Travis WD, Banks PM and Reiman HM: Primary extranodal soft tissue lymphoma of the extremities. Am J Surg Pathol 11: 359-366, 1987.

10. Suresh S, Saifuddin A and O'Donnell P: Lymphoma presenting as a musculoskeletal soft tissue mass: MRI findings in 24 cases. Eur Radiol 18: 2628-2634, 2008.

11. Hwang S: Imaging of lymphoma of the musculoskeletal system. Radiol Clin North Am 46: 379-396, 2008.

12. Damron TA, Le MH, Rooney MT, Vermont A and Poiesz BJ: Lymphoma presenting as a soft tissue mass. A soft tissue sarcoma simulator. Clin Orthop Relat Res 221-230, 1999.

13. Chun CW, Jee WH, Park HJ, Kim YJ, Park JM, Lee SH and Park SH: MRI features of skeletal muscle lymphoma. AJR Am J Roentgenol 195: 1355-1360, 2010.

14. Hwang S: Imaging of lymphoma of the musculoskeletal system. Magn Reson Imaging Clin N Am 18: 75-93, 2010.

15. Kang HJ, Beylergil V,Price AP, Abramson SJ and Carrasquillo JA: FDG PET/CT detection of intussusception caused by lymphoma in a pediatric patient. Clin Nucl Med 39: 97-98, 2014.

16. Knowles B and Serpell JW: Extra-nodal lymphoma presenting as a mimic of soft-tissue sarcoma. ANZ J Surg 73: 26-30, 2003.

17. Coiffier B, Lepage E, Briere J, Herbrecht R, Tilly H, Bouabdallah R, Morel P, Van Den Neste E, Salles G, Gaulard P, et al: CHOP chemotherapy plus rituximab compared with CHOP alone in elderly patients with diffuse large-B-cell lymphoma. N Engl J Med 346: 235-242, 2002.

18. Feugier P, Van Hoof A, Sebban C, Solal-Celigny P,Bouabdallah R, Fermé C, Christian B, Lepage E, Tilly H, Morschhauser F, et al: Long-term results of the R-CHOP study in the treatment of elderly patients with diffuse large B-cell lymphoma: A study by the Groupe d'Etude des Lymphomes de l'Adulte. J Clin Oncol 23: 4117-4126, 2005

19. Habermann TM, Weller EA, Morrison VA, Gascoyne RD, Cassileth PA, Cohn JB, Dakhil SR, Woda B, Fisher RI, Peterson BA, et al: Rituximab-CHOP versus CHOP alone or with maintenance rituximab in older patients with diffuse large B-cell lymphoma. J Clin Oncol 24: 3121-3127, 2006.

20. Coiffier B, Thieblemont C, Van Den Neste E, Lepeu G, Plantier I, Castaigne S, Lefort S, Marit G, Macro M, Sebban C, et al: Long-term outcome of patients in the LNH-98.5 trial, the first randomized study comparing rituximab-CHOP to standard CHOP chemotherapy in DLBCL patients: A study by the Groupe d'Etudes des Lymphomes de l'Adulte. Blood 116: 2040-2045, 2010.

21. Niitsu N: Current treatment strategy of diffuse large B-cell lymphomas. Int J Hematol 92: 231-237, 2010.

22. Spina M,BalzarottiM,Uziel L,Ferreri AJ,FratinoL, Magagnoli M, Talamini R, Giacalone A, Ravaioli E, Chimienti E, et al: Modulated chemotherapy according to modified comprehensive geriatric assessment in 100 consecutive elderly patients with diffuse large B-cell lymphoma. Oncologist 17: 838-846, 2012.

23. Huang Z, Xu Z and Zhou Y: Chemotherapy alone versus chemotherapy followed by consolidative radiotherapy for limited-stage aggressive non-Hodgkin's lymphoma: A meta-analysis of randomized controlled trials. Cancer Radiother 17: 736-743, 2013.

24. Dorth JA, Prosnitz LR, Broadwater G, Beaven AW and Kelsey CR: Radiotherapy dose-response analysis for diffuse large B-cell lymphoma with a complete response to chemotherapy. Radiat Oncol 7: 100, 2012. 
25. Hou Y, Wang HQ and Ba Y: Rituximab, gemcitabine, cisplatin and dexamethasone in patients with refractory or relapsed aggressive B-cell lymphoma. Med Oncol 29: 2409-2416, 2012.

26. Aribi M, Mesli N, Remla N, Sari BE, Taleb A, Touhami H, Bekadja MA, Zouaoui-Benhadji Z, Bouzid K and Meguenni K: Gemcitabine and treatment of diffuse large B-cell lymphoma in relapsed or refractory elderly patients: A prospective randomized trial in Algeria. J Cancer Res Ther 6: 41-46, 2010.
27. Crump M, Baetz T, Couban S, Belch A, Marcellus D, Howson-Jan K, Imrie K, Myers R, Adams G, Ding K, et al: Gemcitabine, dexamethasone, and cisplatin in patients with recurrent or refractory aggressive histology B-cell non-Hodgkin lymphoma: A Phase II study by the national cancer institute of Canada clinical trials group (NCIC-CTG). Cancer 101: 1835-1842, 2004. 